\title{
Les boues des stations d'épuration
}

\author{
Sewage treatment sludge \\ par J. Bontoux \\ Professeur à la Faculté de Pharmacie \\ Université de Montpellier.
}

Sludge production is highly increasing ; their tipping will be borbidden in a near future; agriculturists are incited to accept broad irrigation of sewage.

What are the advantages and the risks for the agriculturists and for the agriculture products consumers ? The advantage is that the fertilizing products are almost free of charge. The risks are the receiver environment alteration or potential health risks.

But in the risks evaluation, subjective dangers perception is often prevalent on the objective risks estimation.

\section{INTRODUCTION}

Les boues des stations d'épuration des eaux usées et leur valorisation en agriculture sont actuellement sujets de débats ; l'opinion publique accepte de plus en plus mal tout ce qui serait une source de risques, d'autant plus que ces risques sont mal cernés.

Les boues sont, sur le plan de la réglementation, des déchets, quelle que soit leur qualité : ce ne sont pas des matières fertilisantes normalisés.

En France, $60 \%$ environ des boues issues des stations d'épuration urbaines sont épandues ( $37 \%$ seulement en Europe). Cet épandage concerne 2 à $3 \%$ de la surface agricole utile en tenant compte de la rotation des terres.

Les 10 millions de tonnes de boues produites $(900000$ tonnes de matières sèches) sont à comparer avec les 2 à 300 millions de tonnes de déjections animales issues des centaines de milliers d'élevages, les 20 millions de tonnes d'ordures ménagères, les 3 à 4 millions de tonnes potentielles de "déchets verts".

On prévoit que la production de boues en France va subir une augmentation de l'ordre de $60 \%$ d'ici 5 ans à cause de l'application des nouvelles directives européennes qui imposent un développement de l'épuration et notamment une meilleure déphosphoration des eaux.

Enfin, à I'horizon 2002, la mise en décharge des boues (25\% des boues françaises, plus de $50 \%$ en Europe, en 1997) doit être interdite ; et les boues aujourd'hui concernées devront être dirigées soit vers l'épandage, soit vers l'incinération.

L'abandon de la valorisation agricole de l'eau entraînerait une très forte augmentation du prix de l'eau.
II $\square$ QUELS AVANTAGES ET QUELS RISQUES REPRÉSENTENT LES BOUES POUR LES AGRICULTEURS ET POUR LES CONSOMMATEURS DES PRODUITS RÉCOLTÉS ?

Les boues ont une valeur agronomique ; elles sont riches en éléments nutritifs pour les cultures : matière organique, azote, phosphore. Ce sont bien, de fait, des substituts des matières fertilisantes dont l'agriculteur adapte l'emploi en fonction des sols et des cultures.

D'autre part les boues sont très souvent chaulées ; l'apport de chaux est un moyen de stabilisation, d'hygiénisation, d'inertage (précipitation des éléments traces métalliques) et de structuration des boues.

L'épandage des boues chaulées est un amendement apprécié gratuit pour l'agriculteur.

La contrepartie de ces avantages réside dans la présence possible de contaminants.

Les contaminants sont tous ceux des eaux usées qui se concentrent facilement dans les boues.

Les contaminants biologiques (bactéries, virus, parasites) représentent des risques épidémiques à court terme ; mais en fait, il n'y a pas de cas prouvé de contamination humaine par les boues d'épuration urbaines. Certains cas litigieux (tuberculose bovine) existent pour les animaux, mais n'ont pu être formellement prouvés : ils seraient de toute façon dus à de mauvaises pratiques d'épandage.

Par contre, quelques rares cas de contaminations de la population par l'épandage d'eaux usées brutes sont connus.

On peut prendre en compte les risques biologiques notamment les risques parasitaires à partir de l'estimation des expositions.

Deux paramètres sont essentiels alors pour l'évaluation 
des risques : les doses minimales infectantes (DMI) et les durées de survie dans l'environnement. On conçoit que les incertitudes à leur propos soient importantes.

Aux contaminants biologiques classiques s'ajoutent aujourd'hui les agents transmissibles non conventionnels (ATNC) responsables des encéphalopathies subaiguës spongiformes transmissibles (ESST) plus connues sous le nom de "maladie de la vache folle".

Le Comité interministériel sur les ESST consulté par le Conseil Supérieur d'Hygiène Publique de France (CSHPF) a préconisé " par mesure de précaution, ... d'éviter le recyclage de produits potentiellement dangereux pour l'alimentation humaine et animale au travers de l'épandage des boues issues des industries d'équarrissage".

Le CSHPF dans son avis publié en septembre 1997 et la nouvelle réglementation française (décret $n^{\circ}$ 97-1133 du 8 décembre 1997 et arrêté du 8 janvier 1998) ne permettent l'épandage des boues brutes qu'à titre tout à fait exceptionnel.

Les boues doivent être traitées afin de maîtriser les risques sanitaires d'ordre biologique grâce à des règles d'usage simples, et seules les boues dites "hygiénisées ", où les concentrations en salmonelles, entérovirus et æufs d'helminthes sont ramenés au-dessous des seuils de sensibilité des méthodes d'analyses de référence, peuvent être épandues sur les cultures de produits pouvant être consommés crus.

Les contaminants chimiques posent des questions de toxicité.

Là encore, seule la voie de la mesure des expositions permet d'évaluer les risques parce que l'épidémiologie est pratiquement aveugle dans le domaine de l'apport des très faibles doses.

Les données toxicologiques sur des éléments traces, que l'on peut trouver dans les boues, sont relativement nombreuses ; des lacunes cependant existent dans la connaissance des conséquences de la "spéciation " sur la toxicité, mais surtout sur les interactions entre les métaux et les divers composants du sol.

Pour les contaminants organiques, les besoins de recherche sont beaucoup plus importants.

Leur identification et leur dosage sont particulièrement difficiles et onéreux. Et les effets toxiques qu'ils peuvent induire, notamment sur les processus de la reproduction (xénoestrogènes), sont actuellement à peu près impossibles à cerner.

Le CSHPF a établi, à titre purement indicatif une liste de molécules susceptibles d'être recherchées dans les boues afin d'élaborer des banques de données, qui à terme devraient conduire à une approche plus rationnelle.

Il demeure de toute façon une différence fondamentale entre éléments traces et contaminants organiques : les éléments traces représentent des contaminations permanentes sous réserve de transferts généralement hydrauliques; les molécules organiques ont des durées de vie limitées dans les sols (demi- vie de l'ordre de quelques dizaines d'années pour les PCB ou les HAP).

Actuellement, les quelques bases réglementaires que l'on trouve dans les différents pays reposent sur des bases scientifiques relativement limitées.

\section{III — LA GESTION DES RISQUES LIÉS À L'ÉPANDAGE}

Elle passe par deux démarches :

- la fixation de valeurs limites de concentration dans les produits épandus,

- la définition de règles d'usage strictes (contrôle des cultures sur les sols recevant les boues, temps à respecter entre épandage et récolte, fixation des distances minimum entre épandage et habitations ou épandage et points d'eau, contrôle des stockages, vêtements spéciaux pour les travailleurs manipulant les boues...).

Les valeurs limites de concentration seront très différentes selon le concept adopté pour les définir.

Il y a deux attitudes extrêmes :

- celle de certaines populations du nord de l'Europe qui n'admettent un apport de contaminants sur les sols qu'autant que cet apport sera exporté par les cultures et que les concentrations dans les sols ne seront pas modifiées. Cette attitude exclut pratiquement l'épandage des boues.

- celle plus classique qui admet des apports de contaminants autant qu'il n'y a pas de risques sanitaires pour les consommateurs des produits récoltés sur les sols recevant les boues. On admet alors une augmentation qui peut être relativement forte des contaminants dans les sols car les coefficients de transfert vers les plantes sont souvent faibles (les facteurs de concentration sont, par exemple, de 0,006 à 0,024 pour l'ensemble des HAP et les racines de carotte; de 0,01 à 0,02 pour le benzo(a)pyrène et les racines de radis et de 0,02 à 0,05 pour le benzo(a)pyrène et les feuilles d'épinards).

Les Etats-Unis et surtout l'Europe ont des démarches intermédiaires en prenant en compte les effets sanitaires possibles non seulement pour l'homme mais aussi sur les différents compartiments biologiques du milieu environnant.

Ainsi des experts de l'Institut Polytechnique de Lausanne ont, dans une étude conduite pour le compte des Agences françaises de l'Eau, décomposé l'environnement en 6 secteurs (eaux souterraines, sol, écosystèmes aquatiques superficiels, végétaux, faune supérieure et homme) : la valeur limite admissible proposée dépend alors du secteur le plus sensible.

En évaluant ainsi les risques, on s'aperçoit que les risques les plus importants pour les animaux et pour l'homme correspondent aux transferts directs à partir du sol et des boues. En période de sécheresse, les absorptions de particules de sols par les animaux sur pâturage peuvent atteindre $10 \%$, ou même plus, de la matière sèche consommée.

\section{IV — LA PERCEPTION SUBJECTIVE DES DANGERS PREND SOUVENT LE PAS SUR L'APPRÉCIATION DES RISQUES OBJECTIFS}

Les risques liés à un épandage bien géré de boues sont extrêmement limités mais comme dans tous les autres domaines, il n'est pas possible d'affirmer que le risque est nul et l'on entre donc dans le débat très difficile du risque acceptable.

Les contraintes imposées aux agriculteurs, qui utilisent les boues, amplifient parfois la perception des risques et elles peuvent entraîner ainsi des situations de blocage qu'il serait souhaitable d'éviter, sinon les boues iront vers des filières très pénalisantes sur le plan économique et probablement plus négatives pour la protection de l'environnement.

\section{BIBLIOGRAPHIE}

[1] Conseil Supérieur d'Hygiène Publique de France. Risques sanitaires liés aux boues d'épuration des eaux usées urbaines. LAVOISIER 1998.

[2] ADEME. I. Feix et J. Wyart. Connaissance et maitrise des aspects sanitaires de l'épandage des boues d'épuration des collectivités locales, FNDAE, 1998

[3] WHO/EOS/95.20.. CHAN A.C., PAGE A.L., TAKASHI A. Developping human health-related chemical guidelines for reclaimed wastewater and sewage sludge application in agriculture. p 114, 1995. 